\title{
ACCELERATION OF PARTICLES BY THE FIELDS OF WAVE PACKETS
}

\author{
V.A. Buts ${ }^{1,2,3}$, V.V. Kuzmin ${ }^{1}$, A.P. Tolstoluzhsky ${ }^{1}$ \\ ${ }^{1}$ National Science Center "Kharkov Institute of Physics and Technology”, Kharkiv, Ukraine; \\ ${ }^{2}$ Institute of Radio Astronomy of NAS of Ukraine, Kharkiv, Ukraine; \\ ${ }^{3}$ V.N. Karazin Kharkiv National University, Kharkiv, Ukraine \\ E-mail:vbuts@kipt.kharkov.ua
}

The dynamics of particles in the field of a wave packet excited in a plasma is considered. The conditions are found under which such dynamics is regular, and when it becomes chaotic. It was found that the well-known (phenomenological) criterion for the emergence of dynamic chaos - the criterion for overlapping Chirikov nonlinear resonances - requires careful use.

PACS: 41.75.-i; 05.45

\section{INTRODUCTION}

When charged particles are accelerated by electromagnetic waves, in particular, when accelerated by laser radiation, electromagnetic fields are mainly modeled by a coherent electromagnetic wave field. In real conditions, the fields are limited both in space and in time (for example, long electromagnetic pulses). Under these conditions, sometimes it is necessary to model such fields with an electromagnetic packet. The question arises: "What features of the dynamics of charged particles can arise in this case and under what conditions is it justified to simulate such a packet by a coherent electromagnetic wave?" This paper answers these questions. The motion of particles in a plasma in the general case obeys random dynamics. This fact is noted when describing the results of numerous theoretical and experimental works (see, for example [1,2]). A rigorous proof of this fact and the criteria for the emergence of regimes with dynamic chaos were obtained for cyclotron resonances (see [3 - 6]). The dynamics of particles in the fields of numerous waves that are excited in plasma should also be chaotic (in the absence of cyclotron resonances). Intuitively, this fact is not in doubt. However, there is apparently no rigorous proof of this fact and the conditions for the occurrence of such dynamics. Below, using simple examples, the conditions have been obtained when this statement is true, as well as the conditions when the particle dynamics left regular.

\section{NON-RELATIVISTIC DYNAMICS OF PARTICLES IN THE FIELDS OF WAVE PACKETS}

It is known that plasma, especially plasma in a magnetic field, has a rich spectrum of natural wave oscillations. Let us show that plasma particles in these even regular fields move randomly. To prove this, consider the movement of charged particles in the field of a wave packet:

$$
\ddot{z}=\frac{e}{m} \sum E_{i} \sin \left(k_{i} z-\omega_{i} t\right) .
$$

Let us first consider the dynamics in the field of a single wave. For such dynamics, from equation (1), we can obtain the well-known integral:

$$
\frac{\dot{\varphi}^{2}}{2}-\Omega^{2} \cos \varphi=H=\text { const },
$$

where $\varphi=k z-\omega t, \Omega^{2}=|e| E k / m \omega^{2}, \dot{\varphi}=d \varphi / d \tau$, $\tau=\omega t$.

Using the integral (2), we find the width of the nonlinear resonance:

$$
\dot{\varphi}_{\max }=+2 \Omega, \dot{\varphi}_{\min }=-2 \Omega .
$$

To determine the distance between resonances, we note that the effective interaction of particles with packet waves occurs under Cherenkov resonance conditions. In this case, it is easy to determine the distance between the resonances:

$$
\Delta \dot{\varphi}=-\Delta k\left[v-\frac{\Delta \omega}{\Delta k}\right] .
$$

Upon receipt of (4), it was taken into account that $v=v_{p h}=\omega / \mathrm{k}$.

Using expressions (3) and (4), we find the conditions for the occurrence of local instability:

$$
K=\frac{4 \Omega}{\Delta \dot{\varphi}}=N \frac{4 \Omega}{\left(1-v_{g} / v_{p h}\right)} \quad K>1,
$$

where $v_{g}$ - group speed; $N=\omega / \Delta \omega-$ the number of waves in the package.

By analyzing formulas (4) and (5), several important conclusions can be drawn. The first one shows (from formula (4)) that if the group velocity tends to the phase velocity of the wave, then the distance between the resonances tends to zero. This means that all the waves of the packet are located in a rectilinear dispersion region. In the phase space, the resonances of such waves all coincide. For particles, such resonances are almost indistinguishable. Dynamics should be regular. Second, if the group wave velocity tends to zero (for example, Langmuir waves in a plasma), then, as can be seen from formula (5) $1<K<<N$; $\Omega<<1$. In this case, as it was the first, apparently, it was noted in [7], the particle dynamics should be chaotic. We note that the dynamics of particles in a plasma almost always corresponds to the case $\Omega<<1$ Indeed, the maximum electric field strength of a longitudinal wave in a plasma (with complete separation of charges) is described by the expression $E_{\text {max }}=\sqrt{4 \pi n m c^{2} \gamma}$.

$$
\Omega^{2}=A=\frac{e E_{\max }}{m \gamma c \omega_{p}}=1,
$$




$$
\left(\omega=\omega_{p}, k=\omega_{p} / c, m \rightarrow m \gamma\right) .
$$

If $K>N$, then the dynamics should be regular. In this case, the particle does not distinguish the resonances of the individual waves of the packet. We are used to the fact that as soon as the inequality $K>1$ is fulfilled, the particle dynamics becomes chaotic. It can be seen that this simple, convenient, and very common criterion requires careful use when it comes to particle dynamics in wave packets.

\section{RELATIVISTIC DYNAMICS OF PARTICLES IN THE FIELDS OF WAVE PACKAGES}

In the relativistic case, the system of equations (1) should be rewritten:

$$
\dot{P}=\sum_{n=1}^{N} A_{n} \sin \psi_{n} \quad \dot{x}=\frac{P}{\gamma} .
$$

The following dimensionless dependent and independent variables are introduced here:

$$
\begin{gathered}
\tau=\omega t, \omega_{n} / \omega, p=\frac{p}{m c}, A_{n}=\frac{e E_{n}}{m c \omega}, \\
\psi_{n}=\omega_{n} \tau-k_{n} z, \tau=\omega t, \quad z=\omega z / c, \\
k_{n}=k_{n} c / \omega=k_{n} / k, k=\omega / c, \dot{p}=d p / d \tau,
\end{gathered}
$$

$\omega$ - maximum frequency of the spectrum of the wave packet.

Multiply the left and right sides of equation (6) by, we obtain a useful equation for the dimensionless particle energy:

$$
\dot{\gamma}=v \cdot \sum_{n=1}^{N} A_{n} \sin \psi_{n},
$$

where $v=v / c, \gamma=\sqrt{1+p^{2}}$.

Below we assume that the momentum of the particle is large $(p>>1)$. In this case, using equations (6) and (7), we can obtain the equation of the mathematical pendulum for the phase of the separately allocated wave of the wave packet:

$$
\ddot{\psi}_{n}+\Omega_{n}^{2}(\tau) \psi_{n}=0,
$$

where $\Omega_{n}^{2}(\tau)=\left[A_{n} / 2 p^{3}\right]$.

Note that the frequency of the mathematical pendulum, which describes the capture vibrations of particles in the wave field, is a function of time. In addition, it is seen that the frequency of these capture vibrations decreases with increasing particle momentum. This dependence is quite obvious, since with increasing momentum the particles become heavier. In full accordance with the algorithm described in the previous section, we can determine the condition for the emergence of a regime with dynamic chaos. This condition can be represented as:

$$
K=\frac{4 \Omega_{n}}{\Delta \dot{\psi}_{n}} .
$$

We note that in both the relativistic and nonrelativistic cases we are talking about Cherenkov resonances. Therefore, the distance between resonances in the relativistic case does not differ from the distance between resonances in the nonrelativistic case $\left(\Delta \dot{\psi}_{n}=\Delta \dot{\varphi}_{n}\right)$.
Finally, the expression for the conditions for the emergence of dynamic chaos takes the form:

$$
K=\frac{4 \Omega_{n}}{\Delta \dot{\psi}_{n}}=N \frac{4 \Omega_{n}}{\left(1-v_{g} / v_{p h}\right)} .
$$

Formula (10) practically does not differ from formula (5). The difference lies only in the physical content of the numerator. From formula (10), one can see the important result that, with an increase in the energy of accelerated particles, the particle dynamics is regularized (parameter $K$ decreases). This result is quite obvious, since with increasing energy the particles become heavier.

Numerical modeling can show a number of features of particle dynamics in the fields of wave packets. Using the example of the relativistic case, we show the dependence of particle dynamics on the ratio of the phase and group velocities of packet waves. To do this, we rewrite the system of equations (6) in the form:

$$
\begin{gathered}
\dot{x}=\frac{x_{1}}{\sqrt{1+x_{1}^{2}}}, \\
\dot{P}=-A \sum_{i=0}^{N} \sin \left[\left(k 1+i \frac{\Delta k}{N}\right) x_{0}-\left(\omega 1+i \frac{\Delta \omega}{N}\right) t\right],
\end{gathered}
$$

where $A$ - amplitude of the waves in the packet; $k 1$, $\omega 1-$ wave vector and frequency of the initial wave; $\Delta k$, $\Delta \omega-$ difference between the wave vectors and the frequencies of the extreme waves of the packet; $N$ - number of waves in the packet.

The initial conditions were chosen so that the particle was in the center of the resonance. $x 0=0$, $P 0=\omega 1 / k 1$. The wavenumbers of the extreme waves of the packet are $\mathrm{k} 1=2, k n=1$, and the frequency of the first wave is also fixed $\omega 1=1$. The waves of the packet were evenly distributed between two fixed waves.

In the case when the group velocity tends to 0 , in this case it is possible when the frequencies of all waves become the same, then the particle dynamics in the field of such a packet turns out to be chaotic Fig. 1 .

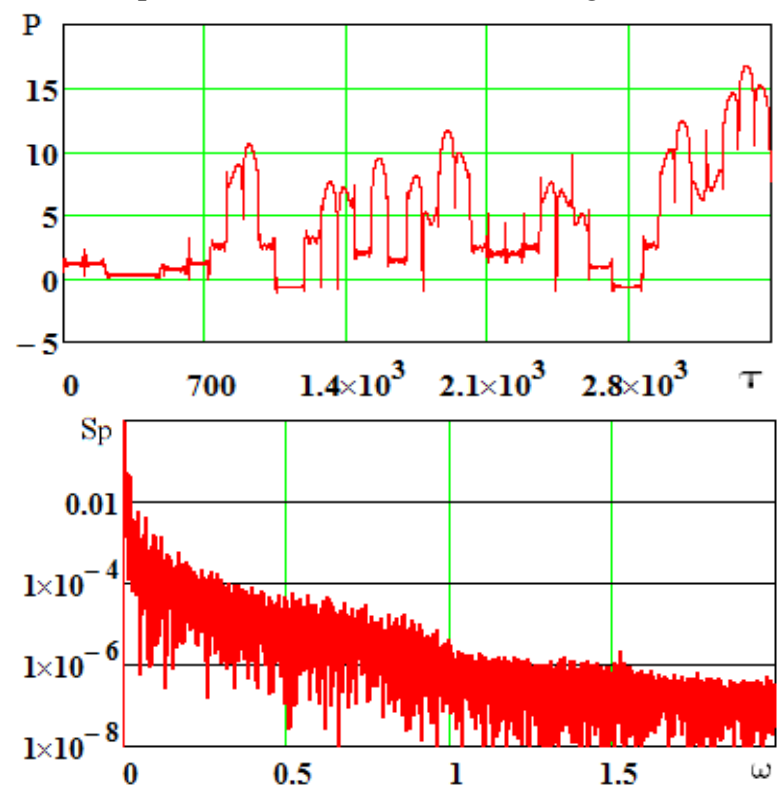

Fig. 1. Particle momentum and its spectrum in the packet field at $v_{g}=0, N=40, A=0.03$ 
In the case when the group velocity tends to the phase velocity, the particle dynamics in the field of such a packet becomes regular see Fig. 2 .
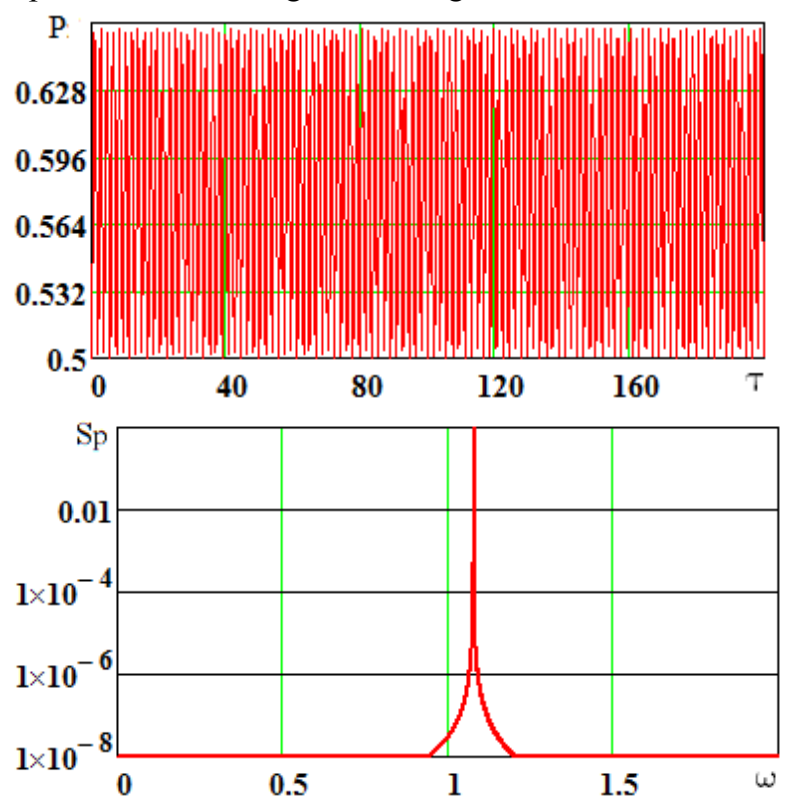

Fig 2. Particle momentum and its spectrum

in the packet field at $v_{g}=v_{p h}, N=40, A=0.03$

It is of interest to consider in more detail the case of regular particle dynamics in a packet. In this case, it can be expected that particles captured in the wave packet will transfer from the field of one wave with a lower phase velocity to the field of another wave with a higher phase velocity. It should be noted that in the existing representation of particle dynamics in the field of several waves, such particle dynamics should be chaotic dynamics. As follows from formula (10), for the case when the number of waves $\mathrm{N}$ is greater than $K(N>K)$, the dynamics will be regular. In this case, one can expect a significant increase in the energy of the particles that interact with this package. Indeed, as follows from the analysis of numerical results (Figs. 3, 4), such a regular particle dynamics in a wave packet is easily realized. These figures show the dependence of the longitudinal momentum of particles on time. Fig. 3 shows the dynamics of particles in a three-wave field, and 4 shows the same dynamics in a 30 -wave field. It can be seen that the dynamics are regular. It corresponds to the dynamics of particles in the field of some effective wave amplitude, which is greater than the amplitudes of the individual waves that make up the package.

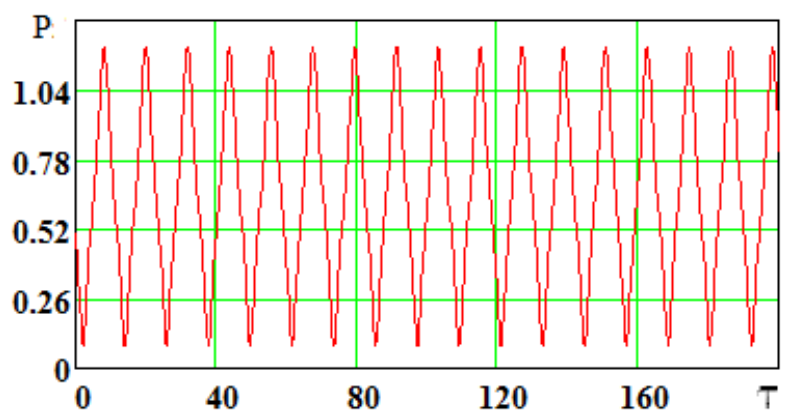

Fig 3. Particle momentum in the packet field at $v_{g}=v_{p h}, N=3, A=0.03$

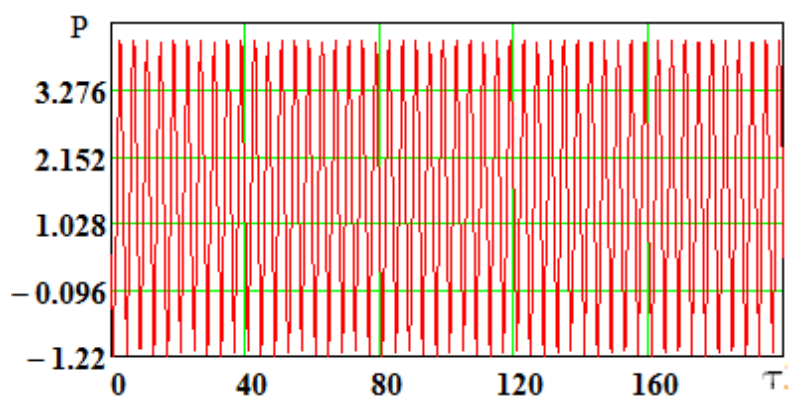

Fig. 4. The particle momentum and its spectrum in the packet field at $v_{g}=v_{p h}, N=40, A=0.03$

Note that for the case of a packet with a large number of waves (30), the maximum value of the longitudinal momentum turns out to be larger than when moving in a packet of 3 waves. This fact is realized, despite the fact that the maximum phase velocity of a wave in a packet of 3 waves is equal to the maximum phase velocity of a wave in a packet of 30 waves. This difference is determined by the fact that the field structure of a wave packet of three waves differs significantly in a packet of 30 waves. This difference is shown in Fig. 5. It can be seen that in a packet of 30 waves the particle experiences periodic intense shocks, the amplitude of which is significantly greater than the amplitudes of individual waves in the packet.

As can be seen from Fig. 5. the maximum value of the force acting on the particles at $N=30$ is an order of magnitude higher than the maximum value of the force at $N=3$.
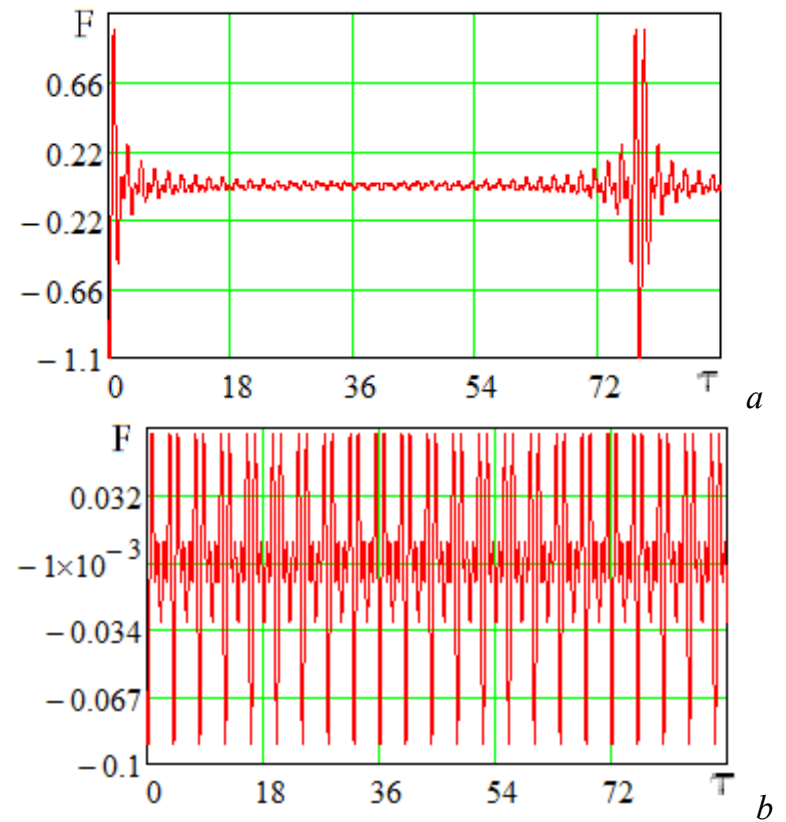

Fig. 5. The force acting on the particle in the field of the wave packet at $N=30(a) ; N=3$ (b)

We emphasize once again that the dynamics remain regular. Note that the dynamics of particles in the field of individual intense pulses (delta function) was studied in detail in numerous works by [7, 8]. Thus, our results show that as soon as $N>K$, the dynamics of particles in such schemes of interaction of waves with particles remains regular.

It is worth noting that a similar dynamics is observed in the nonrelativistic case. 


\section{CONCLUSIONS}

Let's list the most significant results of the work:

1. The well-known wave strength parameter for waves excited in a plasma ( $\left.A=e E / m c \omega \gamma, \omega=\omega_{p}\right)$ does not exceed unity ( $A \leq 1)$.

2 . If the group velocity of the wave packet in the plasma tends to zero, then the particle dynamics in the field of such a packet is chaotic.

3. If the group velocity of the wave packet tends to the phase velocity of the wave packet, then the dynamics of the particles in the wave packet is regular.

4. Analysis of numerical results is in good agreement with analytical results.

5. The dynamics of particles in packets of a small number of waves and the dynamics of particles in a packet of a large number of waves are qualitatively different. However, if the number of waves is greater than the parameter $K(N>K)$, the dynamics in the packet remains regular, even at $\mathrm{K}>>1$.

\section{REFERENCES}

1. E.G. Shustin, N.V. Isaev, M.P. Temiryazeva, V.P. Tarakanov, Yu.V. Fedorov. Beam-Plasma Discharge in a Weak Magnetic Field as a Source of Plasma for a Plasma-Chemical Reactor // Problems of Atomic Science and Technology. 2008, № 4, p. 169.
2. I.N. Meshkov, S.S. Nagaitsev, I.A. Seleznev, E.M. Syresin. Beam - Plasma Discharge at Electron Beam Injection Into Rare Gas: Preprint 90-12. Novosibirsk Institute of Nuclear Physics. 1990, 630090, 15.

3. S.S. Moiseev, V.A. Buts, N.S. Erokhin. Peculiarities of Charged Particle Dynamics under Cyclotron Resonance Conditions // Plasma Physics Reports. 2016, v. 42, № 8, p. 761-768.

4. V.A. Buts, V.V. Kuzmin, A.P. Tolstoluzhsky. Features of the dynamics of particles and fields at cyclotron resonances // ZhETF. 2017, v. 152, № 4 (10), p. 767-780.

5. V.A. Buts, A.N. Lebedev, V.I. Kurilko. The Theory of Coherent Radiation by Intense Electron Beams. Springer, Berlin, 2006.

6. V.A. Buts. Regular and chaotic dynamics of charged particles during wave-particle interactions // Problems of Theoretical Physics. Series "Problems of Theoretical and Mathematical Physics”. 2017, № 2, p. 122-241.

7. G.M. Zaslavsky, B.V. Chirikov. Stochastic instability of nonlinear oscillations // Phys. Usp. 1971, v. 105, p. $1-42$.

8. G.M. Zaslavsky, R.Z. Sagdeev. Introduction to nonlinear physics: from a pendulum to turbulence and chaos. M.: "Nauka", 1988, 368 p.

Article received 23.10.2019

\section{УСКОРЕНИЕ ЧАСТИЦ ПОЛЯМИ ВОЛНОВЫХ ПАКЕТОВ}

\section{В.А. Буи, В.В. Кузьмин, А.П. Толстолужсккий}

Рассмотрена динамика частиц в поле волнового пакета, который возбужден в плазме. Найдены условия, при которых такая динамика является регулярной, и когда она становится хаотичной. Обнаружено, что известный (феноменологический) критерий возникновения динамического хаоса - критерий перекрытия нелинейных резонансов Чирикова, требует осторожного использования.

\section{ПРИСКОРЕННЯ ЧАСТИНОК ПОЛЯМИ ХВИЛЬОВИХ ПАКЕТІВ}

\section{В.О. Буц, В.В. Кузьмін, О.П. Толстолужський}

Розглянута динаміка частинок у полі хвилевого пакету, що збуджений у плазмі. Знайдено умови, при яких така динаміка $є$ регулярною, і коли вона є хаотичною. Виявлено, що відомий (феноменологічний) критерій виникнення динамічного хаосу - критерій перекриття нелінійних резонансів Чирікова, вимагає обережного використання. 